\title{
Relationship between dementia severity in older women and family caregivers' preferences for shared decision making about breast cancer screening
}

\author{
Molly Frank ${ }^{1}$, Nicole Fowler $1,2,3,4$ \\ ${ }^{1}$ Indiana University School of Medicine; ${ }^{2}$ Division of General Internal Medicine and Geriatrics; \\ ${ }^{3}$ Indiana University Center for Aging Research; ${ }^{4}$ Regenstrief Institute, Inc.
}

Background/Objective: Mammography is one of the most effective ways to diagnose breast cancer early; however, its perceived benefits are complicated by terminal conditions such as dementia. By undergoing mammography, women with dementia risk treatment complications and false-positive results, which can exacerbate psychological distress. The lack of a standard of care confounds the individual roles of the patient, family caregiver, and physician in the decision-making process. This study evaluates the relationship between dementia severity and family caregiver preferences for shared decision making.

Methods: Data were gathered from the Decisions about Cancer screening in Alzheimer's Disease trial, which uses the Dementia Severity Rating Scale (DSRS) and a revised version of the Control Preferences Scale (CPS) to assess family caregiver preferences for decisionmaking as a dyad (patient and caregiver) and triad (patient, caregiver, and physician). Two multinomial logistic regression models assessed the relationship between DSRS and CPS categories (active, passive, and collaborative), while controlling for the caregivers' age, gender, education, relationship to patient, self-perceived income, and race. Both models used the "active" group as the baseline; however, Model 1 examined preferences as a dyad and Model 2 as a triad.

Results: Model 1 found a statistically significant association between dementia severity and a collaborative approach $(p<0.001)$, and between dementia severity and a passive approach $(p=0.014)$. For every one-unit increase in DSRS score, the odds of being in the collaborative group decreased by 0.083 and the odds of being in the passive group decreased by 0.085 . There was no statistically significant association between dementia severity and decisionmaking preferences in Model 2.

Clinical Significance: The association between dementia severity and family caregiver decision-making preferences supports the need for a standard of care regarding breast cancer screening in women with dementia. 